\title{
Anatomy vs physiology: Is that the question?
}

\author{
Leslee J. Shaw, PhD \\ ECCRI, Emory University School of Medicine, Atlanta, GA
}

doi: $10.1007 / \mathrm{s} 12350-013-9849-1$

\section{See related article, pp. 284-290}

There is a general tenet in nuclear cardiology that the greater the underlying coronary stenosis, the higher the likelihood of functionally significant myocardial ischemia. This correlation is even stronger in the setting of a significant left main coronary stenosis or triplevessel coronary artery disease (CAD) where the severity of ischemic defects is frequently profound and encumbering a large proportion of the myocardium. It is reported that nearly $95 \%$ of patients with severe left main or 3-vessel CAD will have an abnormal stress myocardial perfusion SPECT. ${ }^{1,2}$ The remainder, with normal stress findings, are the focus of research examining the concept of balanced reduction in myocardial perfusion due to high risk CAD. ${ }^{3,4}$ In the current issue of the journal, Yokota et $\mathrm{al}^{5}$ examines the prevalence of severe left main or its equivalent triple-vessel CAD in the setting of normal stress myocardial perfusion findings. Of 256 symptomatic patients undergoing prompt coronary angiography following a normal stress myocardial perfusion scan, $7 \%$ had left main and $10 \%$ had 3-vessel CAD. These findings are consistent with prior research $^{3}$ and suggest that the false negative rate for a normal stress myocardial perfusion SPECT, albeit a safety concern for most clinicians, is relatively modest. This report also includes prognostic findings in the setting of a normal stress SPECT and report an annual death rate of $\sim 1.7 \%$ in the 93 patients with significant $\mathrm{CAD}$; a rate similar to the remaining 163 patients with nonobstructive CAD. This death rate is consistent with the hundreds of registry series reporting an annual death

Reprint requests: Leslee J. Shaw, PhD, ECCRI, Emory University School of Medicine, 1529 Clifton Rd NE, Rm 529, Atlanta, GA; lshaw3@emory.edu

J Nucl Cardiol 2014;21:291-2.

$1071-3581 / \$ 34.00$

Copyright (C) 2014 American Society of Nuclear Cardiology. rate that is equated to low risk status in those with a normal stress myocardial perfusion SPECT scan. ${ }^{6}$ These low risk prognostic findings suggest that patients with a normal stress myocardial perfusion SPECT scan have few events and, even in the presence of underlying severe CAD, anatomic adaptations, such as collateralization, may be poorly documented but result in normalization of flow and improvement of clinical outcomes for these CAD patients.

In the examination of concordance between functional measurements of ischemia with anatomic lesions, the diagnostic sensitivity and specificity statistics that are frequently published can be misleading since anatomic adaptations to the development of an obstructive lesion can foster collateral development resulting in normal flow rates. These accuracy statistics are even more misleading in patients with prior revascularization where mechanical intervention normalizes blood flow to one or more subtended myocardial regions. These statistics identify false negative rates based on the presence of an obstructive lesion in the setting of normal stress myocardial perfusion findings to be approximately $10 \% .^{6}$ The ancillary anatomic findings which may explain the discordance between a normal physiologic response to stress and severe obstructive CAD are rarely documented. However, these cases illustrate the complexity and interplay of factors underlying physiologic flow states and atherosclerotic disease extent and severity; factors which in their complexity cannot be illustrated by a single statistic. Increasingly, multivariable models or complex risk scores illustrate the multifaceted nature of pathophysiologic disease states and may help to put forth an improved understanding of the atherosclerotic disease process.

However, the occurrence of balanced reduction in flow due to severe CAD is a safety concern for high likelihood patients. Several reports identify non-perfusion, electrocardiographic, or calcified plaque location and extent as well as additional non-perfusion findings that may improve the detection of significant CAD in the setting of normal stress myocardial perfusion SPECT results. $^{3,4,7,8}$ A careful review of ancillary findings 
including prior test results as well as ECG stress risk markers can provide clues as to the presence of underlying CAD in the setting of normal stress SPECT results. Moreover, the extensiveness of peripheral atherosclerosis and adaptations to a patient's symptomatic burden and demand ischemia can result in reduced physical work capacity and the documentation of submaximal exercise levels on the index stress test. These factors serve to illustrate that interpreting nuclear cardiology procedures is difficult and requires a high level of advanced training in order to provide high quality interpretive services to our patients. Too often, the procedures are interpreted based solely on the perfusion imaging parameters and fail to incorporate electrocardiographic, arrhythmic, clinical, and other ancillary markers which can substantially improve the quality of diagnostic and prognostic interpretation. The documentation of severe CAD at the time of coronary angiography in patients with a recent normal stress myocardial perfusion SPECT scan can erode the confidence levels of referring physicians including the interventionalists. Similar discordance is also documented between invasive fractional flow reserve and CAD stenosis. Nuclear cardiologists should wisely engage in the heart team discussions with regards to interventional cardiology practices and provide an expansive reservoir of knowledge on the complex interplay between anatomic and physiologic disease states in order to provide nuclear cardiology services that optimally serve to enhance diagnostic acumen, guide patient care, and improve patient outcomes.

\section{References}

1. Iskandrian AS, Heo J, Nguyen T, Beer SG, Cave V, Ogilby JD, et al. Assessment of coronary artery disease using single-photon emission computed tomography with thallium-201 during adenosine-induced coronary hyperemia. Am J Cardiol 1991;67:1190-4.

2. Beller GA, Zaret BL. Contributions of nuclear cardiology to diagnosis and prognosis of patients with coronary artery disease. Circulation 2000;101:1465-78.

3. Berman DS, Kang X, Slomka PJ, Gerlach J, de Yang L, Hayes SW, et al. Underestimation of extent of ischemia by gated spect myocardial perfusion imaging in patients with left main coronary artery disease. J Nucl Cardiol 2007;14:521-8.

4. Maddahi J, Abdulla A, Garcia EV, Swan HJ, Berman DS. Noninvasive identification of left main and triple vessel coronary artery disease: Improved accuracy using quantitative analysis of regional myocardial stress distribution and washout of thallium201. J Am Coll Cardiol 1986;7:53-60.

5. Yokota S, Ottervanger JP, Mouden M, Timmer JR, Knollema S, Jager PL. Prevalence, location, and extent of significant coronary artery disease in patients with normal myocardial perfusion imaging. J Nucl Cardiol. doi:10.1007/s12350-013-9837-5.

6. Shaw LJ, Hage FG, Berman DS, Hachamovitch R, Iskandrian A. Prognosis in the era of comparative effectiveness research: Where is nuclear cardiology now and where should it be? J Nucl Cardiol 2012;19:1026-43.

7. Valenta I, Dilsizian V, Quercioli A, Ruddy TD, Schindler TH. Quantitative PET/CT measures of myocardial flow reserve and atherosclerosis for cardiac risk assessment and predicting adverse patient outcomes. Curr Cardiol Rep 2013;15:344.

8. Ghadri JR, Pazhenkottil AP, Nkoulou RN, Goetti R, Buechel RR, Husmann L, et al. Very high coronary calcium score unmasks obstructive coronary artery disease in patients with normal SPECT MPI. Heart 2011;97:998-1003.

\section{Disclosure}

There are no disclosures relevant to this editorial. 